\title{
Clima organizacional y su relación con el desempeño laboral de los docentes universitarios, Chachapoyas, 2019
}

\section{Organizational climate and its relationship with the work performance of university teachers, Chachapoyas, 2019}

\author{
Estefany Salazar Grandez ${ }^{1}$
}

\section{RESUMEN}

Esta investigación se trazó el objetivo de determinar la relación existente entre el clima organizacional y el desempeño laboral de los docentes de la Escuela Profesional de Ciencias de la Comunicación de la Universidad Nacional Toribio Rodríguez de Mendoza de Amazonas (UNTRM), Perú, en el año 2019. La metodología empleada fue de tipo descriptivo-correlacional y manejó como instrumento la encuesta. La población muestral estuvo constituida por 17 docentes activos. Se encontró que un $64.7 \%$ de los profesores perciben un buen clima organizacional y un $17.6 \%$ como excelente; con respecto al desempeño laboral docente, se encontró que un 52,9\% presentó un excelente desempeño laboral y el 5,9\% muy deficiente. Se concluye que existe una correlación positiva baja $(+)$ entre las variables desempeño laboral y clima organizacional, ya que su correlación es Rs $=+0.261$, con una significancia $\mathrm{p}=0.312>0.05$.

Palabras claves: clima organizacional, desempeño laboral

\begin{abstract}
This research aimed to determine the relationship between the organizational climate and the work performance of the teachers of the Professional School of Communication Sciences of the National University Toribio Rodríguez de Mendoza de Amazonas (UNTRM), Peru, in the year 2019. The methodology used was descriptive-correlational and used the survey as an instrument. The population and sample consisted of 17 active teachers. It was found that $64.7 \%$ of the teachers perceive a good organizational climate and $17.6 \%$ as excellent; With regard to teaching work performance, it was found that $52.9 \%$ of the teachers evaluated have excellent work performance and $5.9 \%$ very poor. It is concluded that there is a low positive correlation $(+)$ between the variables work performance and organizational climate, since its correlation is $\mathrm{Rs}=+0.261$, with a significance $\mathrm{p}=0.312>0.05$.
\end{abstract}

Keywords: organizational climate, job performance

${ }^{1}$ Bachiller en Comunicación Social. Escuela Profesional de Ciencias de la Comunicación, Facultad de Educación y Ciencias de la Comunicación de la Universidad Nacional Toribio Rodríguez de Mendoza de Amazonas. Correo estefany.salazar@untrm.edu.pe 


\section{INTRODUCCIÓN}

El desempeño laboral docente toma un papel protagónico en la calidad de la formación de profesionales que año tras año, se vienen forjando en las aulas de las diferentes casas superiores de estudio. Las acciones que vienen desarrollando los docentes al momento de impartir sus conocimientos, muchas veces se ven afectadas por diversos factores, dentro de ellos, el clima organizacional del lugar donde desempeñan; si éste no es el adecuado, trae consigo diversas consecuencias, dentro de ellas, un déficit en la enseñanza brindada y, por ende, una formación profesional con bajos estándares de calidad con respecto a la educación superior.

Bajo este argumento, podemos afirmar el rol fundamental que cumple el clima organizacional en el desempeño laboral, en especial, adentrándonos hacia nuestra realidad, Magallán (2018) al referirse a la Universidad Nacional Toribio Rodríguez de Mendoza de a Amazonas (UNTRM) indica que al igual que en las demás instituciones tanto públicas como privadas, el clima organizacional se ve afectado por diversos problemas de carácter interno y externo, en este caso, factores que tienen incidencia directa en los servicios educativos, los cuales se hacen evidentes en reuniones con docentes, administrativos y estudiantes, quienes muestran su descontento, considerando que éste presenta una intervención directa en el ejercicio docente y como consecuencia, en la enseñanza de los educandos universitarios.

Es por ello que en la Escuela Profesional de Ciencias de la Comunicación de la UNTRM en su constante necesidad de cumplir con el objetivo de obtener la acreditación y alcanzar la excelencia académica, debe someterse a un proceso constante de cambio, en beneficio de la misma, persiguiendo como principal meta que la educación impartida por los docentes cumpla con los estándares de calidad. De este modo, el clima organizacional se considera un elemento sustancial en el desarrollo de este proceso en beneficio del desempeño docente; de esta variable dependerá el cumplimiento eficiente de las funciones que desempeñan al momento de impartir sus conocimientos. Debido a esto, se consideró de vital relevancia ejecutar un estudio orientado en determinar la relación que existe entre nuestras variables de estudio. Al mismo tiempo, este estudio forma una base teórica para realizar aportación de conocimientos a futuras pesquisas concernientes al clima organizacional y desempeño laboral, otorgando así, mayores argumentos que coadyuvarán a efectuar pesquisas asemejadas a nuestra realidad.

Para identificar la realidad en la que nos encontramos, se buscaron antecedentes de soporte para la presente investigación, las cuales contaban con variables semejantes; las que, sin embargo, por su naturaleza, difieren de la investigación. Villadiego y Alzate (2017) en su investigación buscó determinar la relación existente entre el desempeño laboral y las relaciones interpersonales del recurso humano y el clima organizacional. Trabajó con una muestra de 8 trabajadores, siendo ésta $100 \%$ de la población. Se manejó el cuestionario, donde fueron analizadas diferentes variables de mucha relevancia en el ambiente laboral de la organización. La pesquisa demostró que el desempeño laboral no presenta mucha relación en el clima, en balance con otras, como la variable de las relaciones interpersonales que sí intervienen ampliamente y no se hallan sólidas, donde conviene concretar procedimientos para disminuir los problemas.

Asimismo, Magallán (2018) en la elaboración de su tesis, planteó el objetivo de determinar el valor de dominio del clima organizacional en el desempeño laboral de los catedráticos de la UNTRM, ejecutada con una muestra establecida por 250 profesores. Finalmente, concluye afirmando el alto grado de dominio del clima organizacional en el desempeño docente. A su vez, Delgado (2019) en la ejecución de su tesis cuyo objetivo fue diagnosticar el clima organizacional y la satisfacción laboral de los profesores nombrados de la UNTRM, llevó a cabo su evaluación aplicándose una encuesta en las 8 facultades de la universidad, con un total de 95 maestros. Cuyas conclusiones demostraron la evidente relación entre estas dos variables, demostrándose que si el clima organizacional cuenta con las condiciones apropiadas dentro de sus distintas dimensiones, de igual modo, será mayor el desempeño laboral del personal.

En consecuencia, nos planteamos el objetivo general de determinar la relación entre el clima organizacional y el desempeño laboral de los docentes de la Escuela Profesional de Ciencias de la Comunicación de la Universidad Nacional Toribio Rodríguez de Mendoza de Amazonas, 2019.

\section{MATERIAL Y MÉTODO}

Diseño de investigación: el diseño empleado fue el descriptivo correlacional.

\section{Población, Muestra y Muestreo}

La población muestral fue compuesta por 17 docentes de la Escuela Profesional de Ciencias de la Comunicación de la UNTRM.

\section{Métodos, técnicas e instrumentos de recolección de datos}

Se emplearon como instrumento de recolección de datos dos encuestas denominados: Encuesta del clima organizacional y Encuesta de desempeño laboral de los docentes (Magallán,2018), las que 
fueron modificadas a las condiciones de la investigación.

La encuesta del clima organizacional estuvo constituido por 28 ítems en escala Likert de 1-5 puntos de nivel de acuerdo: totalmente de acuerdo (5), de acuerdo (4), ni en acuerdo ni en desacuerdo (3), en desacuerdo (2) y totalmente en desacuerdo (1), para sus tres dimensiones. La encuesta sobre desempeño laboral consta 30 ítems en escala Likert de 1-5 puntos de valoración como fueron: siempre (5), casi siempre (4), a veces (3), casi nunca (2), nunca (1). Estos ítems se estructuraron en sus tres dimensiones las cuales implicaron 7 indicadores.

El instrumento se validó por juicio de expertos, la confiabilidad se realizó con prueba piloto y se calculó con el indicador estadístico Alfa de Cronbach, donde se logró un índice de confiabilidad de 0,934 sobre la encuesta del clima organizacional y de 0,960 sobre la encuesta que evaluó el desempeño laboral.

\section{RESULTADOS}

\section{Figura 1}

Distribución porcentual del clima organizacional en la Escuela Profesional de Ciencias de la Comunicación de la UNTRM, 2019

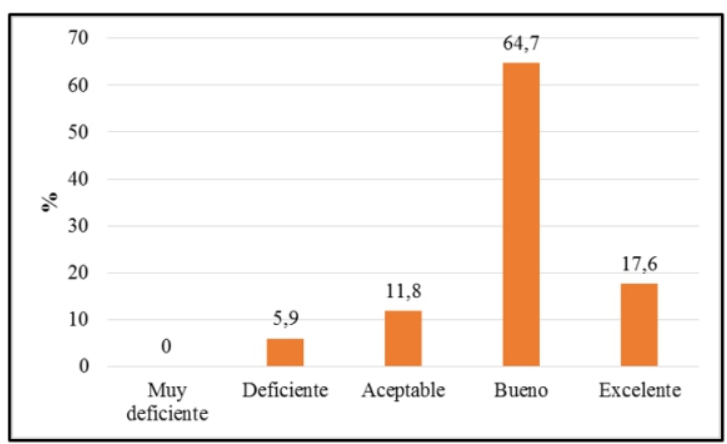

En la figura 1, se evidencia que el 64,7\% de docentes tuvieron una percepción de buen nivel en el clima organizacional, $17,6 \%$ nivel excelente; y 5,9\% de nivel deficiente, lo que significa que el clima organizacional en la E.P. Ciencias de la Comunicación 2019, se caracterizó por ser de nivel bueno en mayor proporción.

\section{Figura 2}

Distribución porcentual de la dimensión cultura organizacional de la variable clima organizacional la Escuela Profesional de Ciencias de la Comunicación de la UNTRM, 2019

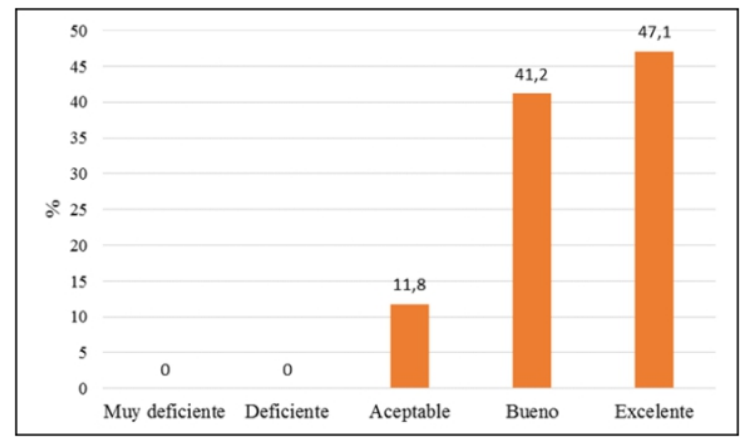

En la figura 2, se encontró que un 47,1\% de profesores tuvieron excelente percepción de la dimensión cultura organizacional, $41,2 \%$ de nivel bueno; y $11,8 \%$ de nivel aceptable, lo que significa que se caracterizó por ser de nivel excelente en mayor proporción.

\section{Figura 3}

Distribución porcentual de la dimensión diseño organizacional de la variable clima organizacional la Escuela Profesional de Ciencias de la Comunicación de la UNTRM, 2019

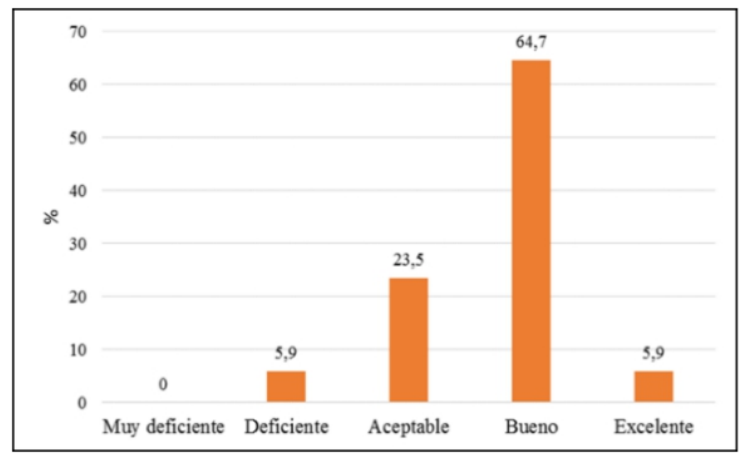

En la figura 3, se encontró que un $64,7 \%$ de profesores tuvieron buena percepción en cuanto a la dimensión diseño organizacional; el 23,5\% la percibieron como aceptable; el 5,9\% excelente, mientras que el 5,9\% restante tuvo una percepción de deficiente; lo que significa que la dimensión diseño organizacional se caracterizó por ser de buen nivel en mayor proporción. 


\section{Figura 4}

Distribución porcentual de la dimensión potencial humano de la variable clima organizacional la Escuela Profesional de Ciencias de la Comunicación de la UNTRM, 2019

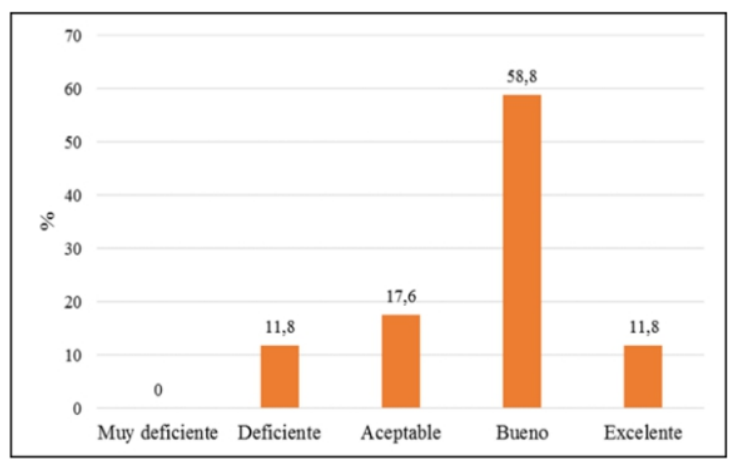

En la figura 4, se encontró que el 58,8\% de la muestra tuvo una buena percepción con respecto a la dimensión potencial humano; el 17,6\% la percibieron como aceptable; el 11,8\% excelente, mientras que el 11,8\% restante tuvo una percepción de deficiente; lo que significa que el potencial humano se caracterizó por ser de buen nivel en su mayoría.

\section{Figura 5}

Distribución porcentual del nivel de desempeño laboral docente en la Escuela Profesional de Ciencias de la Comunicación de la UNTRM, 2019

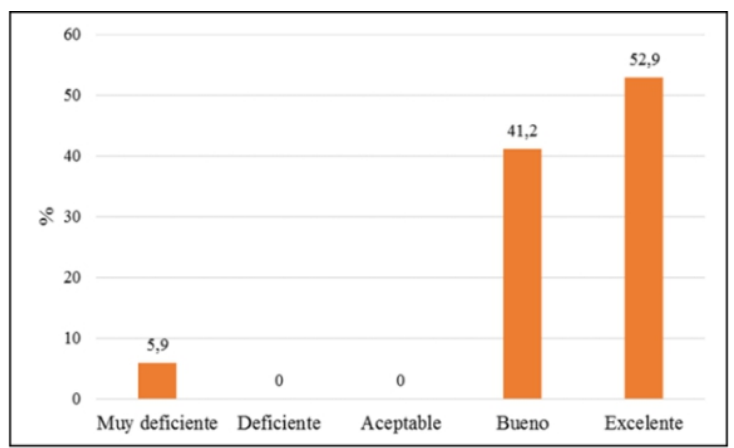

En la figura 5, se observa que un 52,9\% tuvieron un nivel excelente en cuanto al desempeño laboral, $41,2 \%$ bueno y $5,9 \%$ deficiente, lo que evidencia mayor proporción con un nivel excelente en esta variable.

\section{Figura 6}

Distribución porcentual de la dimensión docencia de la variable desempeño laboral docente en la Escuela Profesional de Ciencias de la Comunicación de la UNTRM, 2019

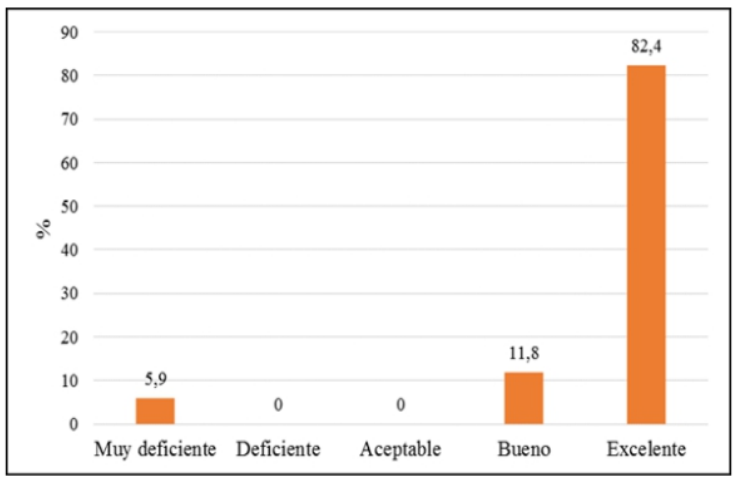

En la figura 6, se encontró que el 82,4\% de docentes tuvieron un nivel excelente en la dimensión docencia, $11,8 \%$ bueno y $5,9 \%$ deficiente, lo que evidencia mayor proporción con un nivel excelente en la dimensión docencia.

\section{Figura 7}

Distribución porcentual de la dimensión investigación de la variable desempeño laboral docente en la Escuela Profesional de Ciencias de la Comunicación de la UNTRM, 2019

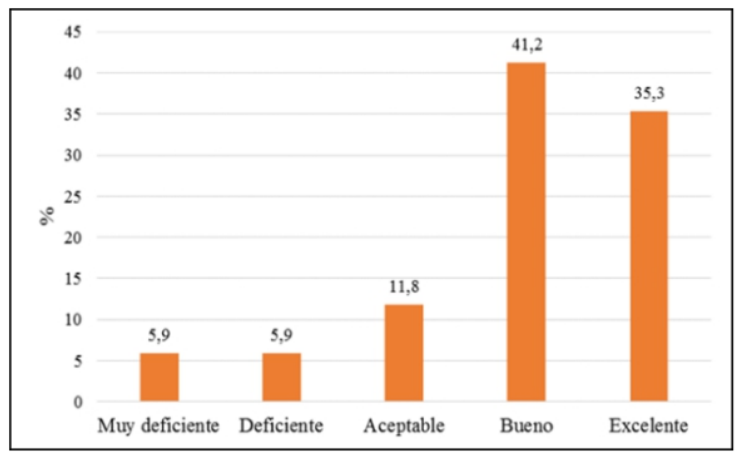

En la figura 7, se encontró un 41,2\% de profesores que tuvieron un nivel bueno en la dimensión investigación, 35,3\% excelente, 11,8 aceptable, 5,9 deficiente y $5,9 \%$ restante fue muy deficiente, lo que evidencia un nivel bueno en mayor proporción en la dimensión docencia. 


\section{Figura 8}

Distribución porcentual de la dimensión proyección de la variable desempeño laboral docente en la Escuela Profesional de Ciencias de la Comunicación de la UNTRM, 2019

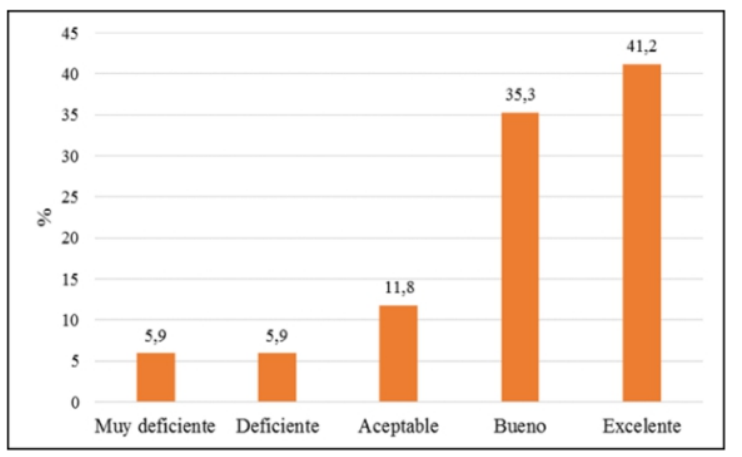

En la figura 8 , fue demostrado que $41,2 \%$ de profesores tuvieron una puntuación excelente en la dimensión proyección y extensión universitaria de la variable desempeño laboral, $35,3 \%$ bueno, 11,8 aceptable, 5,9 deficiente y 5,9\% restante fue muy deficiente, lo que evidencia un nivel excelente en mayor proporción en la dimensión docencia.

\section{Tabla 1}

Valores de correlación de las variables desempeño laboral y clima organizacional según dimensiones

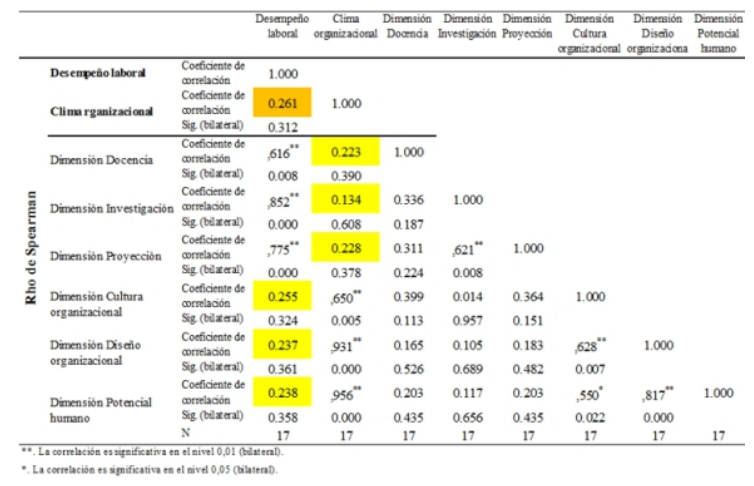

En la tabla 1, se evaluó la correlación entre el desempeño laboral y clima organizacional en los docentes de la Escuela Profesional de Ciencias de la Comunicación de la UNTRM, donde, según la tabla de categorías, se hace evidente una correlación positiva baja $(+) R s=+0.261$, con una significancia $\mathrm{p}=0.312>0.05$. De igual manera, al evaluar las correlaciones bivariados de las variables y sus dimensiones se puede evidenciar correlaciones positivas bajas.

\section{DISCUSIÓN}

Los resultados encontrados en nuestra investigación revelan una correlación positiva baja $(+) \mathrm{Rs}=+0.261$ entre el desempeño laboral y clima organizacional, presentando una significancia $p=0.312>0.05$. De igual manera, al evaluar las correlaciones bivariados de las variables y sus dimensiones se puede evidenciar correlaciones positivas bajas. A su vez, hallamos que un $64,7 \%$ obtuvo una percepción buena nivel en clima organizacional y que, el 52,9\% tuvieron un nivel excelente en cuanto a desempeño laboral, lo que evidencia mayor proporción con un nivel excelente en dicha variable. Los resultados en mención fueron contrastados con Delgado (2019) donde encontró una relación positiva entre el clima organizacional y la satisfacción laboral, donde si existen condiciones óptimas para el clima organizacional y sus dimensiones, el desempeño laboral será más favorable. Entonces, ambas tesis de investigación comparten resultados semejantes; es decir, los docentes que perciben clima organizacional bueno, tendrán a su vez, un buen desempeño docente.

En diversas actividades, nuestro desempeño en muchas veces se ve influenciado por factores externos, tales como el clima laboral que percibimos, por lo que, si no estamos conformes con nuestro entorno laboral, hay mayores probabilidades que nuestro desempeño se vea afectado. Los resultados encontrados en nuestra investigación arrojan que el $64,7 \%$ de profesores percibieron un buen clima organizacional, $17,6 \%$ nivel excelente; y 5,9\% de nivel deficiente, lo que significa que se caracterizó por ser de nivel bueno en mayor porcentaje. En contraste con lo expuesto, Zans (2017) encontró que el clima organizacional, en mayor proporción fue determinado como optimista, a diferencia de que en cuanto a frialdad y distanciamiento en un porcentaje menor, el cual es interpretado en que si se presenta la existencia de un mejor clima organizacional, aportaría éste a su vez, de forma beneficiosa al desempeño laboral. Además de la semejanza de variables, se comparten resultados semejantes, donde se evidencia que el $64,7 \%$ de la muestra percibe un buen clima organizacional en mayor proporción.

Con respecto a la a la dimensión cultura organizacional, obtuvimos un $47,1 \%$ de docentes que tuvieron una percepción excelente, favoreciendo así, su desempeño laboral; sin embargo, nuestros resultados obtenidos divergen con la investigación desarrollada por Villadiego y Alzate (2017) donde su pesquisa demostró que el desempeño laboral afecta en fuerte proporción en el clima, pero que a pesar de ello, se deben realizar aportes y soluciones para prevenir que éste signifique un riesgo. Sin embargo, son los tratos laborales los que sí intervienen con mayor proporción y que, al mismo tiempo, no se encuentran consolidadas entre los trabajadores; finalizando afirmando que las variables estudiadas no se encuentran directamente relacionadas.

Asimismo, en cuanto al diseño organizacional se determinó que el $64,7 \%$ de docentes tuvieron una buena percepción, encontrándose similitudes con la pesquisa desarrollada por Magallán (2018), donde 
además de contar con las mimas variables de estudio, afirma un índice elevado de influjo del clima organizacional en el desempeño laboral, resultados que tienen cierta similitud con los arrojados por nuestra investigación, en el cual es evidente la relación positiva entre nuestras variables estudiadas, con una relación relativamente menor a los obtenidos en la investigación desarrollada.

Por otra parte, refiriéndonos a la dimensión potencial humano; el 17,6\% la percibieron como aceptable; el $11,8 \%$ excelente, mientras que el $11,8 \%$ restante tuvo una percepción de deficiente; lo que significa que se caracterizó por ser de buen nivel en mayor proporción, donde coincidimos parcialmente con la investigación desarrollada por Ramos (2018) donde se encontró que en cuanto a los índices del desarrollo de la profesionalidad, el $66,67 \%$ de los profesores presentó un valor medio, el 25,29\% un valor bajo y el $8,05 \%$ un alto valor; afirmando de este modo que, los docentes en su mayoría evidencian un valor medio en cuanto a desarrollo de sus competencias profesionales y la identidad como docentes; siendo estos resultados, similares a los nuestros.

Del mismo modo, en cuanto al desempeño laboral, Barriga (2016) encontró que la aptitud humana se encuentra relacionada con el desempeño docente, al igual que el clima, la cultura y el diseño organizacional. Resultados que comparten mucha semejanza con los nuestros, donde se encontró que el $52,9 \%$ de los docentes tuvieron una puntuación excelente en desempeño laboral, relacionados con la cultura organizacional, diseño organizacional y potencial humano.

En cuanto a las dimensiones docencia y proyección social y extensión universitaria, nuestros resultados arrojaron ser de nivel excelente en mayor proporción, teniendo un porcentaje de $82,4 \%$ y $41,2 \%$ respectivamente, hallándose semejanzas a los encontrados por Reyes (2017) cuyos resultados determinaron la relación directa y significativa entre el clima organizacional y el desempeño docente, entendiéndose el gran influjo que atribuye el clima organizacional en el desempeño laboral.

Con respecto a la investigación, se encontró buen nivel con un porcentaje de $41,2 \%$ en mayor proporción, encontrándose similitudes con la pesquisa desarrollada por Magallán (2018) quien considera que la dimensión investigación es un aspecto que se tiene que mejorar, al mismo tiempo como la docencia y la proyección social, debiéndose aplicar teorías pedagógicas, didácticas y curriculares, seguido de usar metodologías para el proceso enseñanza, además el servicio a la sociedad $\mathrm{y}$, investigar en la cátedra, diseñar, elaborar y aplicar instrumentos de evaluación, además de formular proyectos para la solución de problemas realizando e incentivando la investigación.

Sim embargo, es importante reconocer, que al correlacionar nuestras dos variables de estudio, algunas investigaciones muestran resultados diferentes a los obtenidos por nosotros. Tal como lo mencionamos, en el presente informe de tesis se pudo comprobar que, al evaluar la correlación entre el desempeño laboral y clima organizacional en los docentes de la Escuela Profesional de Ciencias de la Comunicación de la UNTRM, se evidencia una correlación positiva baja $(+)$ con una correlación $R s=+0.261$, y una significancia $p=0.312>0.05$. De igual manera, al evaluar las correlaciones bivariados de las variables y sus dimensiones se puede evidenciar correlaciones positivas bajas, afirmando que el clima organizacional y el desempeño laboral de los docentes están relacionados; hallazgos que difieren con Villadiego y Alzate (2017) donde su investigación finalizó afirmando que el desempeño laboral no demuestra mayor influencia en el clima organizacional.

Finalmente, concluimos este apartado, expresando que nuestros resultados al obtenerse en un solo contexto y en una sola medición, no pueden ser extrapolados a la toda la docencia de UNTRM, o en otras realidades de la región o del país, tomando en cuenta que pese a haberse realizado en otros contextos, los resultados pueden ser similares a los nuestros.

\section{CONCLUSIONES}

El $41,2 \%$ de profesores tuvieron buen nivel en investigación, el 35,3\% excelente, 11,8 aceptable, 5,9 deficiente y $5,9 \%$ restante fue muy deficiente, lo que evidencia un nivel bueno en mayor proporción en la dimensión docencia.

El 41,2\% de docentes presentó una excelente dimensión proyección y extensión universitaria de la variable desempeño laboral, 35,3\% bueno, 11,8 aceptable, 5,9 deficiente y 5,9\% restante fue muy deficiente, evidenciando un nivel excelente en mayor proporción en la dimensión docencia.

Las correlaciones bivariados de las variables y sus dimensiones son positivas bajas con un promedio de 0.11-0.49 en el coeficiente Rho de Spearman entre cada variable y sus dimensiones.

\section{REFERENCIAS BIBLIOGRÁFICAS}

Barriga, R. (2016). Clima organizacional y desempeño docente en la universidad "Jaime Bausate y Meza” Jesús María - Lima, 2016. (Tesis de maestría, Universidad Nacional Mayor de San Marcos). https://core.ac.uk/ download/pdf/323350037.pdf

Delgado, M. (2019). Clima organizacional y satisfacción laboral de los docentes nombrados de la de la Universidad Nacional Toribio 
Rodríguez de Mendoza de Amazonas, Perú 2019. (Tesis de licenciatura, Universidad Nacional Toribio Rodríguez de Mendoza de Amazonas). https://cutt.ly/djDDv1L

Magallán, N. (2018). El clima organizacional y su influencia en el desempeño laboral de los docentes de la Facultad de Ciencias Económicas y Administrativas de la Universidad Nacional Toribio Rodríguez de Mendoza de Amazonas, Chachapoyas 2017-II. (Tesis de licenciatura, Universidad Nacional Toribio Rodríguez de Mendoza de Amazonas). http://repositorio.untrm.edu.pe/handle/UNTR M/1398

Ramos, E. (2018). Clima organizacional y desempeño docente en la institución educativa $N^{\circ} 6038$, Ugel 01, 2018. (Tesis de maestría, Universidad César Vallejo). https://cutt.ly /wjDSnge

Reyes, J. (2017). Clima organizacional y desempeño docente en la Institución Educativa Los Libertadores, Ayacucho, 2013. (Tesis de maestría, Universidad Nacional de Educación Enrique Guzmán y Valle). https://bit.ly/ 30cUhwI

Villadiego, A y Alzate, K. (2017). Análisis del clima organizacional y su relación con el desempeño laboral y las relaciones interpersonales en Petroleum \& Logistics S.A.S. en la ciudad de Bogotá, durante el primer semestre de 2016. (Tesis de licenciatura, Universidad de Cartagena). https://bit.ly/2wfodZh

Zans, A. (2017). Clima Organizacional y su incidencia en el desempeño laboral de los trabajadores administrativos y docentes de la Facultad Regional Multidisciplinaria de Matagalpa, UNAN - Managua en el periodo 2016 (Tesis de maestría, Universidad Nacional Autónoma de Nicaragua). https://bit.ly/2H FzZW1 